\title{
Reactivation of TB during administration of durvalumab after chemoradiotherapy for non-small-cell lung cancer: a case report
}

\author{
Yasuhiro Kato*,1,2, Yasutaka Watanabe1, Yuki Yamane1 ${ }^{1}$, Hideaki Mizutani ${ }^{1}$, Futoshi \\ Kurimoto ${ }^{1}$ \& Hiroshi Sakai ${ }^{1}$ \\ ${ }^{1}$ Department of Thoracic Oncology, Saitama Cancer Center, Saitama, Japan \\ ${ }^{2}$ Department of Pulmonary Medicine and Oncology, Graduate School of Medicine, Nippon Medical School, Tokyo, Japan \\ *Author for correspondence: Tel.: +81 048722 1111; y-kato@nms.ac.jp
}

Background: Tuberculosis (TB) is considered to be an adverse effect of treatment with immune checkpoint inhibitors. Methodology \& results: Our case was a 75-year-old woman diagnosed with unresectable stage III non-small-cell lung cancer. After radical chemoradiotherapy was completed, durvalumab was initiated as a consolidation therapy. However, since chest CT showed appearances of infiltration shadows scattered in the periphery of the lungs after five doses of immunotherapy, duruvalumab was discontinued. 6 weeks later, the patient was aware of intermittent fever. Chest CT scan showed the appearance of a tree-in-bud pattern in the right lung. Acid-fast bacilli stain of sputum was positive and the PCR test was positive for Mycobacterium tuberculosis. Conclusion: Duruvalumab as PD-L1 blockade may activate TB.

First draft submitted: 16 March 2020; Accepted for publication: 1 April 2020; Published online:

21 April 2020

Keywords: chemoradiotherapy • durvalumab • IGRA • immune checkpoint inhibitor • irAE • LTBI • NSCLC • PD-L1 blockade • pulmonary TB • TB

\section{Background}

Immune checkpoint inhibitors (ICIs) have become generalized anticancer agents in the treatment of all types of cancer. Non-small-cell lung cancer (NSCLC) is one of the most common cancers in the world. Many previous trials reported that ICIs provided clinical benefit in patients with NSCLC [1-5]. Therefore, ICIs are considered to be a useful treatment option for NSCLC.

Conversely, various immune-related adverse events have been reported following ICI treatment. Recently, several articles have reported that ICI may lead to the development of active TB [6-15]. However, the causal relationship between ICI and TB remains controversial. Here, we report a case of reactivation of TB following durvalumab treatment after chemoradiotherapy for unresectable stage III NSCLC.

\section{Case presentation}

The case reported here was a 75 -year-old woman, a former smoker ( 45 packs per year) who quit smoking 10 years ago. The patient was referred to our hospital because of lower-limb paralysis, back pain and an abnormal chest shadow on chest x-ray in May 2019. A $67 \mathrm{~mm}$ tumor with cavity, calcification and direct invasion of the chest wall and thoracic spine was revealed in the right upper lobe by chest computed tomography (CT) (Figure 1A-C). In addition, CT showed calcification of the mediastinal lymph nodes and tracheal wall (Figure 1D). Although she did not remembered the details, she had been treated for TB as a teenager. However, the result of initial T-SPOT.TB as an interferon-gamma release assay (IGRA) at the first visit our hospital was negative. Squamous cell carcinoma was detected by trans-bronchial lung biopsy performed for the diagnosis of the tumor, with no targeted gene mutations and over $50 \%$ of programmed death-ligand 1 (PD-L1) expression. She was diagnosed with NSCLC cT4N0M0 stage IIIA after brain MRI and bone scintigraphy revealed the absence of metastatic lesions in the brain and bones. She received radical chemoradiotherapy (weekly carboplatin [AUC2] plus paclitaxel $\left[40 \mathrm{mg} / \mathrm{m}^{2}\right.$ ] with concurrent thoracic radiotherapy at a daily dose of 2 Gy for a total dose of 66 Gy) for unresectable NSCLC. She experienced grade 3 lymphopenia, grade 2 leukopenia, neutropenia, anemia and hypoalbuminemia as adverse 


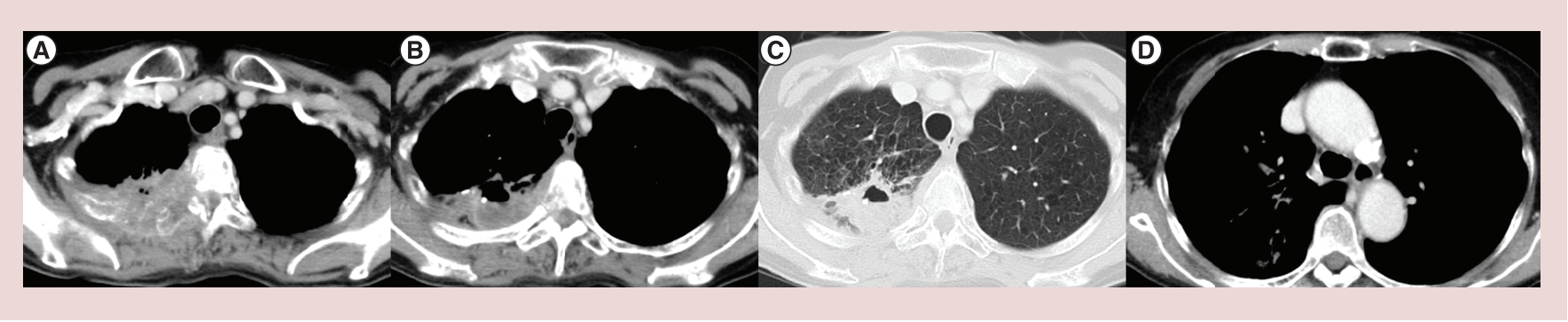

Figure 1. CT images acquired at the diagnosis of stage III non-small-cell lung cancer in our case. (A) Primary non-small-cell lung cancer lesion at the right upper lobe of the lung with invasion of the chest wall and thoracic spine. (B \& C) Primary non-small-cell lung cancer lesion at the right upper lobe of the lung presenting with cavity and calcifications. (D) Calcification of the mediastinal lymph nodes and tracheal wall.

\begin{tabular}{|c|c|c|c|c|c|}
\hline & $\begin{array}{l}\text { At the start of } \\
\text { chemoradiation therapy }\end{array}$ & $\begin{array}{l}\text { After } 6 \text { weeks from the } \\
\text { start of chemoradiation } \\
\text { therapy }\end{array}$ & $\begin{array}{l}\text { After } 9 \text { weeks from the } \\
\text { start of chemoradiation } \\
\text { therapy (at the start of } \\
\text { duruvalumab) }\end{array}$ & $\begin{array}{l}\text { After } 25 \text { weeks from the } \\
\text { start of chemoradiation } \\
\text { therapy (at the } \\
\text { durvalumab } \\
\text { discontinuation) }\end{array}$ & $\begin{array}{l}\text { After } 33 \text { weeks from the } \\
\text { start of chemoradiation } \\
\text { therapy (at the diagnosis of } \\
\text { TB) }\end{array}$ \\
\hline $\operatorname{WBC}\left(10^{3} / \mu \mathrm{l}\right)$ & 12240 & 2330 & 5640 & 5340 & 8190 \\
\hline $\operatorname{Neu}\left(10^{3} / \mu \mathrm{l}\right)$ & 5090 & 1620 & 4460 & 3900 & 6880 \\
\hline $\operatorname{Lym}\left(10^{3} / \mu \mathrm{l}\right)$ & 2130 & 440 & 540 & 830 & 730 \\
\hline $\mathrm{Ht}(\%)$ & 32.1 & 24.7 & 28.4 & 31.1 & 26.1 \\
\hline $\mathrm{Hb}(\mathrm{g} / \mathrm{dl})$ & 10.3 & 8.0 & 9.2 & 9.7 & 8.1 \\
\hline Plt $\left(10^{3} / \mu \mathrm{l}\right)$ & 30.5 & 18.4 & 37.1 & 15.3 & 46.5 \\
\hline
\end{tabular}

events of chemoradiotherapy. Table 1 shows her blood count including lymphocyte count of clinical courses. Her clinical symptoms, such as lower-limb paralysis and back pain, improved significantly and chest CT showed tumor shrinkage after chemoradiotherapy (Figure 2A-D). Biweekly durvalumab treatment was started 2 weeks after the end of the radiation therapy. Bone marrow suppression including anemia and lymphopenia gradually recovered during duruvalumab administration. (Table 1) At the time of fifth course of this therapy, chest CT revealed a reduction of the primary lesion, but showed the appearance of small infiltration shadows scattered in the periphery of both lungs (Figure 2E \& F). She had no subjective symptoms. Durvalumab was discontinued and CT was performed again 1 month later, because of the possibility of the development of interstitial lung disease as an immune-related adverse events. This chest CT showed the presence of enlarged infiltration shadows in both lungs, including the areas around the primary lesion (Figure 2G \& H). We recommended bronchoscopy for the diagnosis of this condition, but the patient rejected this diagnostic modality because of its invasiveness and requested follow-up. However, 2 weeks later she was referred to our hospital because she was aware of an intermittent fever of $38-39^{\circ} \mathrm{C}$ and night sweats. Chest CT showed enlargement of the cavity of the primary lesion, with infiltrate shadows surrounding the primary lesion. In addition, a tree-in-bud pattern was apparent in the right middle and inferior lobes (Figure 2I \& J). T-SPOT.TB and Ziehl-Neelsen staining of a sputum smear sample were positive at this time point. Finally, sputum samples were positive for Mycobacterium tuberculosis (Mtb) DNA, as assessed using PCR. She was diagnosed with reactivation of TB and was transferred to our hospital, where isolation control was conducted. Currently, she continues anti-TB medication and her pulmonary TB is controlled by medication. Her lung cancer is controlled without recurrence despite of anticancer treatment discontinuation.

\section{Discussion}

Several previous reports have described a risk of developing TB during programmed death 1 (PD-1) blockade treatment [6-15]. However, to the best our knowledge, this manuscript was the first report of TB reactivation during durvalumab therapy after radical chemoradiotherapy for stage III NSCLC. 

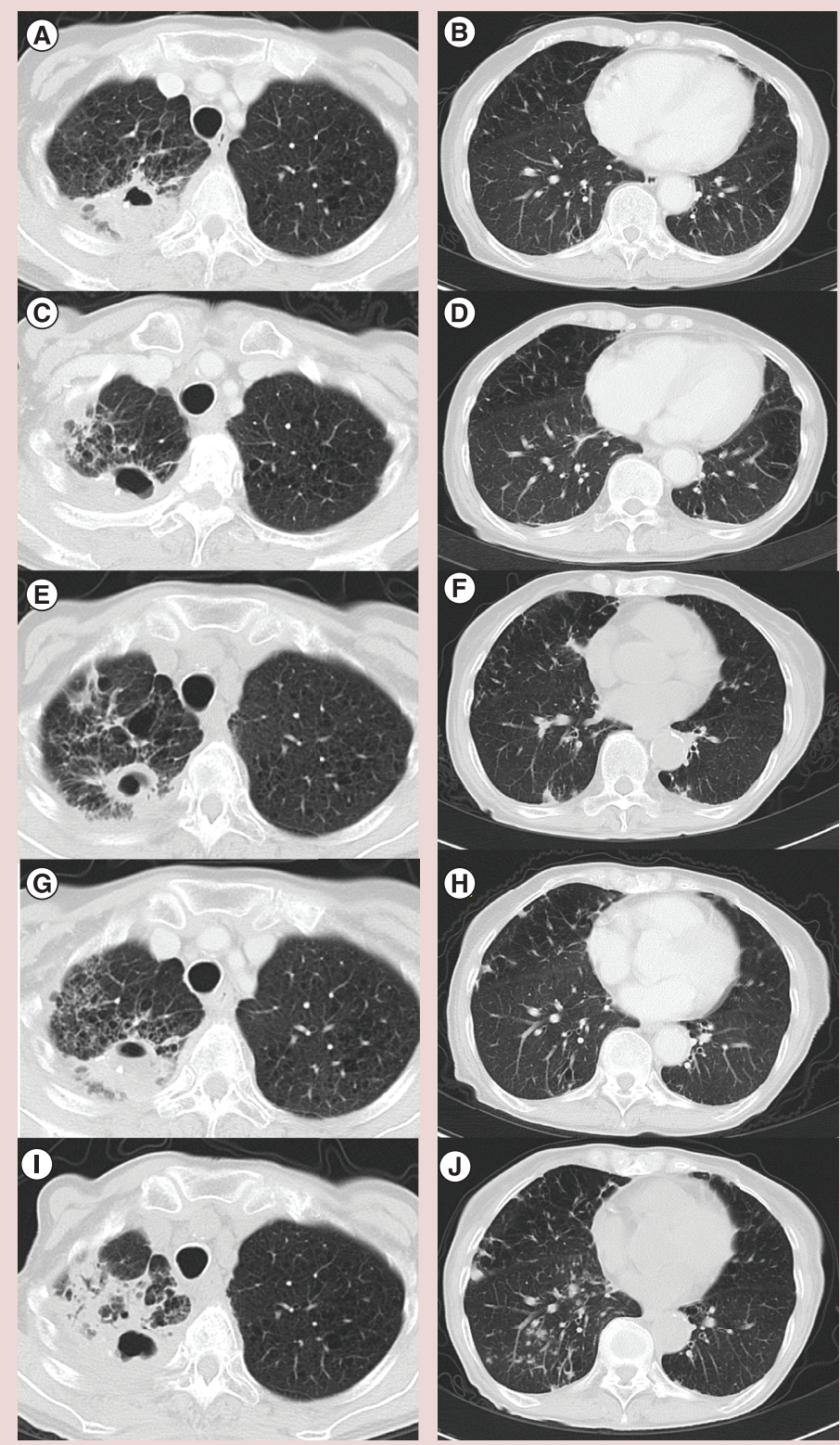

Figure 2. CT images acquired during the clinical course of our case. (A \& B) CT images acquired at the diagnosis of non-small-cell lung cancer. (C \& D) The primary lesion exhibited shrinkage after chemoradiotherapy. (E \& F) CT images acquired after five courses of durvalumab therapy. Although the primary lesion showed further reduction, the appearance of new infiltration shadows scattered in the periphery of both lungs was noted. Therefore, durvalumab was discontinued. (G \& H) CT images acquired 1 month after durvalumab was discontinued. Chest CT showed enlarged infiltration shadows in both lungs, including the areas around the primary lesion. (I \& J) CT images acquired at the diagnosis of active pulmonary TB. Infiltration shadows surrounding the primary lesion and a tree-in-bud pattern in the right inferior lobe were observed.

Durvalumab is an ICI that was developed as a human IgG1 monoclonal antibody that inhibits the binding of PDL1 to PD-1 and CD80. The international PACIFIC Phase III trial, which compared durvalumab with placebo after chemoradiotherapy using platinum doublet for stage III unresectable NSCLC, showed that the progression-free survival and overall survival were longer with durvalumab versus placebo treatment $[16,17]$. Therefore, durvalumab consolidation therapy after radical chemoradiotherapy is currently considered as a standard therapy for unresectable stage III NSCLC. 
PD-1 blockade (nivolumab and pembrolizumab) is considered a risk for the development of active TB. However, this relationship remains controversial. According to preclinical data, PD-1 knockout mice are hypersensitive to Mtb infection, develop large necrotic lesions with a high bacterial load, and exhibit earlier death compared with T-cell deficient mice [18]. Other research has shown that PD-1 knockout mice develop uncontrolled TB because of increased Mtb-specific CD4 T cell (especially TH1 cells) and IFN- $\gamma$ response [19,20]. These results suggest that PD-1 blockade promotes the reactivation of TB by eliciting an excessive immune response against myobacterium. In addition, increased $\mathrm{T}$ regulatory cells and decreased TB-antigen-specific $\mathrm{T}$ cells were observed in PD-1 knockout mice [21]. These findings indicate that the appropriate immune response is inhibited by PD-L1 blockade. Moreover, our case suggests that the same mechanism causes the activation of TB during treatment with durvalumab, which represents a PD-L1 blockade.

Interestingly, our case had a history of TB treatment and the imaging results showed several calcified lesions (the primary lesion, the mediastinal lymph nodes and the tracheal wall), which is characteristic of old pulmonary TB, despite the initial negative IGRA. IGRA detection of the immune response to specific Mtb antigens has good sensitivity and specificity for diagnosing latent tuberculosis infection (LTBI) [22]. In particular, the sensitivity and specificity of T-SPOT.TB for diagnosing LTBI are high [22]. In contrast, previous research reported an IGRA reversion rate of $5.2-22 \%$ [23]. Therefore, we considered our case to have LTBI with reactivated TB infection caused by durvalumab. In addition, it is suggested that LTBI represents a risk of reactivation of TB during immunotherapy.

This work had several limitations. Our case received chemotherapy before durvalumab administration, which may have affected TB activation. In particular, our case experienced grade 3 lymphopenia during chemoradiotherapy. There is no denying that the immunosuppressive state may have affected the reactivation of TB. However, we consider that the effects of chemotherapy were small, because 4 months passed since the start of durvalumab until the diagnosis of TB and the course of chest CT imaging. As Table 1 showed, anemia and lymphopenia caused by chemoradiotherapy was recovered during durvalumab administration, though not completely. In contrast, in relation to the hypothesis of the reactivation of $\mathrm{TB}$ during immunotherapy mentioned above, the immunosuppressive state that was caused by grade 3 lymphopenia reactivated the latent TB, with PD-L1 blockade worsening this reactivated TB state by eliciting an excessive immune response against TB. Further case accumulation and analysis are needed to confirm the hypothesis formulated for our single case.

\section{Conclusion}

In conclusion, we experienced a case of reactivation of $\mathrm{TB}$ following durvalumab treatment after chemoradiotherapy for stage III NSCLC. PD-L1 blockade may have the potential to reactivate latent TB infection.

\section{Summary points}

- Several case reports have demonstrated that immune checkpoint inhibitors have a potential risk of reactivation of latent tuberculosis infection (LTBI).

- A 75-year-old woman was diagnosed with unresectable stage III NSCLC in 2019. She had a medical history of pulmonary tuberculosis (TB) at an early age. After radical chemoradiotherapy (weekly carboplatin [AUC2] plus paclitaxel $\left[40 \mathrm{mg} / \mathrm{m}^{2}\right.$ ] with concurrent thoracic radiotherapy at a daily dose of $2 \mathrm{~Gy}$ and total dose of $66 \mathrm{~Gy}$ ) was completed, durvalumab (at a dose of $10 \mathrm{mg} / \mathrm{kg} /$ body intravenous drip every 2 weeks) administration was initiated as a consolidation therapy.

- In 2019, after five doses of immunotherapy, computed tomography (CT) scanning of the chest showed small infiltration shadows scattered in the periphery of both lungs were observed, despite the shrinkage of the primary lesion.

- Since we considered the development of interstitial lung disease as an immune-related adverse events of durvalumab, durvalumab was discontinued.

- 1 month after durvalumab discontinuation, enlarged infiltration shadows were apparent on chest CT in both lungs, including the areas surrounding the primary lesion, despite the absence of subjective symptoms.

- 2 weeks later, she became aware of intermittent fever and night sweats. Chest CT showed the appearance of a tree-in-bud pattern in the right middle and inferior lobes and of enlargement of the infiltrate shadows surrounding the primary lesion.

- Acid-fast bacilli stain of sputum was positive and the PCR test was positive for Mycobacterium tuberculosis.

- Currently, she continues anti-TB medication, whereas anti-lung cancer treatment is still withheld.

- Durvalumab consolidation treatment after radical chemoradiotherapy for stage III NSCLC has the potential risk of reactivation of LTBI. 
Financial \& competing interests disclosure

H Sakai and H Mizutani received honoraria from AstraZeneca. The authors have no other relevant affiliations or financial involvement with any organization or entity with a financial interest in or financial conflict with the subject matter or materials discussed in the manuscript apart from those disclosed.

The authors would like to thanks Enago for the English language editing services (www.enago.jp).

\section{Ethical conduct of research}

We obtain informed consent from the patient for the publication of this case report and associated images. All procedures performed in studies involving human participants were in accordance with the ethical standards of the institutional and/or national research committee and with the 1964 Helsinki declaration and its later amendments or comparable ethical standards.

\section{Open access}

This work is licensed under the Attribution-NonCommercial-NoDerivatives 4.0 Unported License. To view a copy of this license, visit http://creativecommons.org/licenses/by-nc-nd/4.0/

\section{References}

Papers of special note have been highlighted as: $\bullet$ of interest; $\bullet \bullet$ of considerable interest

1. Borghaei H, Paz-Ares L, Horn L et al. Nivolumab versus docetaxel in advanced nonsquamous non-small-cell lung cancer. N. Engl. J. Med. 373(17), 1627-1639 (2015).

2. Brahmer J, Reckamp KL, Baas $\mathrm{P}$ et al. Nivolumab versus docetaxel in advanced squamous-cell non-small-cell lung cancer. N. Engl. J. Med. 373(2), 123-135 (2015).

3. Mok TSK, Wu YL, Kudaba I et al. Pembrolizumab versus chemotherapy for previously untreated, PD-L1-expressing, locally advanced or metastatic non-small-cell lung cancer (KEYNOTE-042): a randomized, open-label, controlled, phase 3 trial. Lancet 393(10183), 1819-1830 (2019).

4. Reck M, Rodriguez-Abreu D, Robinson AG et al. Pembrolizumab versus chemotherapy for PD-L1-positive non-small-cell lung cancer. N. Engl. J. Med. 375(19), 1823-1833 (2016).

5. Rittmeyer A, Barlesi F, Waterkamp D et al. Atezolizumab versus docetaxel in patients with previously treated non-small-cell lung cancer (OAK): a Phase III, open-label, multicentre randomized controlled trial. Lancet 389(10066), 255-265 (2017).

6. Anastasopoulou A, Ziogas DC, Samarkos M, Kirkwood JM, Gogas H. Reactivation of tuberculosis in cancer patients following administration of immune checkpoint inhibitors: current evidence and clinical practice recommendations. J. Immunother. Cancer 7(1), 239 (2019)

-. Case report and literature review for activated tuberculosis during immune checkpoint inhibitors (ICIs) treatment. It reviewed latent tuberculosis (TB) infection screening including IFN- $\gamma$ release assay for cancer patients. Author suggested the necessity for investigation of usefulness of latent tuberculosis infection (LTBI) screening before ICI.

7. Chu YC, Fang KC, Chen HC et al. Pericardial tamponade caused by a hypersensitivity response to tuberculosis reactivation after anti-PD-1 treatment in a patient with advanced pulmonary adenocarcinoma. J. Thorac. Oncol. 12(8), e111-e114 (2017).

- Case of hypersensitivity response after starting anti-TB treatment for reactivation during anti-programmed death 1 treatment. Author considered immunotherapy affected hype sensitivity response to TB by activation of immunity.

8. Elkington PT, Bateman AC, Thomas GJ, Ottensmeier CH. Implications of tuberculosis reactivation after immune checkpoint inhibition. Am. J. Respir. Crit. Care Med. 198(11), 1451-1453 (2018).

-• Case report of TB during programmed death 1 blockade. Author presented programmed death-ligand 1 and CD8 expression within TB granuloma. It suggested pathway affected by ICI is activated in TB granuloma.

9. Fujita K, Terashima T, Mio T. Anti-PD1 antibody treatment and the development of acute pulmonary tuberculosis. J. Thorac. Oncol. 11(12), 2238-2240 (2016).

- One of the most important articles, because this case report was the first case about activated TB during ICI.

10. He W, Zhang X, Li W et al. Activated pulmonary tuberculosis in a patient with melanoma during PD-1 inhibition: a case report. Onco Targets Ther. 11, 7423-7427 (2018).

11. Jensen KH, Persson G, Bondgaard AL, Pohl M. Development of pulmonary tuberculosis following treatment with anti-PD-1 for non-small-cell lung cancer. Acta Oncol. 57(8), 1127-1128 (2018).

12. Lee JJ, Chan A, Tang T. Tuberculosis reactivation in a patient receiving anti-programmed death-1 (PD-1) inhibitor for relapsed Hodgkin's lymphoma. Acta Oncol. 55(4), 519-520 (2016).

13. Picchi $\mathrm{H}$, Mateus $\mathrm{C}$, Chouaid $\mathrm{C}$ et al. Infectious complications associated with the use of immune checkpoint inhibitors in oncology: reactivation of tuberculosis after anti PD-1 treatment. Clin. Microbiol. Infect. 24(3), 216-218 (2018). 
14. Takata S, Koh G, Han Y et al. Paradoxical response in a patient with non-small-cell lung cancer who received nivolumab followed by anti-Mycobacterium tuberculosis agents. J. Infect. Chemother. 25(1), 54-58 (2019).

15. Tsai CC, Chen JH, Wang YC, Chang FY. Re-activation of pulmonary tuberculosis during anti-programmed death-1 (PD-1) treatment. QJM 112(1), 41-42 (2019).

16. Antonia SJ, Villegas A, Daniel D et al. Overall survival with durvalumab after chemoradiotherapy in stage III NSCLC. N. Engl. J. Med. 379(24), 2342-2350 (2018).

- A landmark paper for unresectable stage III non-small-cell lung cancer (NSCLC) proved consolidation therapy of durvalumab efficacy after chemoradiotherapy.

17. Antonia SJ, Villegas A, Daniel D et al. Durvalumab after chemoradiotherapy in stage III non-small-cell lung cancer. N. Engl. J. Med. 377(20), 1919-1929 (2017).

- Durvalumab showed improvement of median overall survival data of stage III unresectable NSCLC in this paper. This research compiled previous research that presented durvalumab efficacy for unresectable stage III NSCLC.

18. Lazar-Molnar E, Chen B, Sweeney KA et al. Programmed death-1 (PD-1)-deficient mice are extraordinarily sensitive to TB. Proc. Natl Acad Sci. USA 107(30), 13402-13407 (2010).

19. Barber DL, Mayer-Barber KD, Feng CG, Sharpe AH, Sher A. CD4 T cells promote rather than control TB in the absence of PD-1-mediated inhibition. J. Immunol. 186(3), 1598-1607 (2011).

-• This research paper showed PD-1 knockout mice cannot control TB infection because PD-1 knockout mice cannot suppress CD4 T-cell expansion.

20. Sakai S, Kauffman KD, Sallin MA et al. CD4 T cell-derived IFN- $\gamma$ plays a minimal role in control of pulmonary Mycobacterium tuberculosis infection and must be actively repressed by PD-1 to prevent lethal disease. PLoS Pathog. 12(5), e1005667 (2016).

-. This research paper showed PD-1 suppresses the accumulation of parenchymal CD4 T cells and their IFN- $\gamma$ production in Mycobacterium tuberculosis infection. It suggested PD-1 blockade has the possibility of activating TB.

21. Tousif S, Singh Y, Prasad DV, Sharma P, Van Kaer L, Das G. T cells from programmed death-1 deficient mice respond poorly to Mycobacterium TB infection. PLoS ONE 6(5), e19864 (2011).

22. Nienhaus A, Ringshausen FC, Costa JT, Schablon A, Tripodi D. IFN- $\gamma$ release assay versus tuberculin skin test for monitoring TB infection in healthcare workers. Expert Rev. Anti Infect. Ther. 11(1), 37-48 (2013).

23. Zwerling A, Van Den Hof S, Scholten J, Cobelens F, Menzies D, Pai M. IFN- $\gamma$ release assays for tuberculosis screening of healthcare workers: a systematic review. Thorax 67(1), 62-70 (2012). 